\title{
EXPLOTACIÓN Y USO DE MARMORA MALACITANOS EN ÉPOCA ROMANA
}

\author{
EXPLOITATION AND USE OF MARMORA FROM ROMAN MALAGA
}

por

JOSÉ BELTRÁN FORTES y MARÍA LUISA LOZA AZUAGA

RESUMEN Planteamos el estado actual de la investigación sobre la explotación y uso de los principales marmora de época romana procedentes de los actuales territorios malacitanos: los mármoles blancos de la sierra de Mijas/Alpujala-Blanca y las calizas blancas y blanco-rojizas ("rojizo Torcal") de las sierras de la depresión intrabética. Nos fijamos en la localización de las canteras antiguas, el período de explotación y el uso de tales materiales. Finalmente se trata la problemática de la presencia de tales marmora en el llamado "edificio de sillares" de c/Argote de Molina (Sevilla), por su datación en el s. II a.C.

\begin{abstract}
This paper studies the more important roman marmora in the province of Málaga: the white marble of the Mijas/Alpujala-Blanca's mountains and the white and white-red limestones ("rojizo Torcal") of interior mountains. It analyzes the site of the roman stone quarries, the date of exploitation and the use of these stones. At last, it focuses the doubtful presence of those stones of Málaga in the site of c/Argote de Molina (Sevilla) during the II century B.C.
\end{abstract}

\section{O. INTRODUCCIÓN}

La participación en fecha reciente en el II Congreso de Historia Antigua de Málaga (Beltrán, Loza e.p.) nos ha obligado a replantear relativamente "viejas cuestiones", a propósito de los estudios que realizamos hace algo más de un decenio sobre la explotación y uso en época romana -durante los ss. I-III d.C.- de los mármoles blancos malacitanos de las sierras actuales de Mijas y Alpujala-Blanca (Loza 1984-85; Loza 1985; Loza, Beltrán 1990). No obstante, las propias limitaciones impuestas en el marco del citado Congreso, temáticas (ya que estaba dedicado al tema monográfico de comercio y comerciantes en la Antigüedad) y, lógicamente, de extensión de nuestra aportación, nos impidieron desarrollar entonces una serie de cuestiones que tratan de forma más concreta sobre aspectos del fenómeno productivo, analizando el período de explotación y empleo de tales mármoles en época romana. Aunque en ese ámbito se hacen indispensables las propias 
referencias a la comercialización de los productos -elaborados, semielaborados o en bruto-, evitaremos en lo posible un desarrollo amplio de aquéllas, remitiendo, pues, a las Actas del citado Congreso.

Plantearemos asimismo en esta ocasión la comparación de la producción y empleo de los mármoles blancos con los otros marmora malacitanos más importantes que se explotaron en época romana: las calizas blancas y las blanco-rojizas que afloran en diversas zonas de todo el surco intrabético malagueño, desde la serranía de Ronda -al oeste- hasta la depresión de Archidona -al este-, con núcleos importantes entre los que destacan, en primer lugar, el de la sierra del Torcal de Antequera ${ }^{1}$ (fig. 1, A) y, en segundo lugar, el de la sierra de Cañete (fig. 1, B).

Desde una perspectiva general que afecte a toda la Península Ibérica los actuales territorios malacitanos tuvieron una importante representación de centros de explotación pétrea, tanto por la calidad de los productos en el panorama peninsular, como por las condiciones especiales de explotación y comercialización a nivel regional, constituyéndose como adecuados paradigmas a la hora de estudiar tales procesos (cf. ahora Mayer, Rodá 1998). Así, por ejemplo, Canto (1977-78: 181) indicaba que la "caliza blanca antequerana" es la de mayor calidad entre las calizas hispanas, y para Cisneros (1988: 98ss.) el mármol blanco malagueño tuvo una enorme extracción, que avalaría su importancia en el ámbito peninsular y una probable propiedad imperial del conjunto de esas canteras.

Dejando aparte otras explotaciones pétreas de piedras locales, como areniscas o calizas que tienen un aprovechamiento restringido, en función de las necesidades constructivas de una ciudad concreta o incluso de asentamientos no urbanos, nos centraremos en aquellos tipos pétreos que disfrutaron de una explotación importante y un empleo no estrictamente local, ya que ofrecen mayores posibilidades a la hora de entroncarlos con los mecanismos generales de explotación/uso de los marmora en época romana. Nos referimos, en concreto, a los mármoles blancos de las sierras de Mijas y Alpujala-Blanca y a las calizas blancas y blancorojizas del surco intrabético, que recorre la actual provincia malagueña de SO a NE, con un punto central en la sierra del Torcal. A pesar del diferente nivel de conocimientos que tenemos sobre ambos materiales en época antigua, analizaremos de forma paralela los dos ejemplos para precisamente resaltar las similitudes y diferencias que pueden ofrecernos.

De entrada debe constatarse que, en ambos casos, se hace necesario un importante trabajo de prospección, que identifique los posibles restos de explotaciones antiguas aún conservadas, aunque un problema básico estriba en la posible destrucción de los frentes de explotación antiguos debido a la continuidad de las labores extractivas. Dejando aparte actuaciones residuales o esporádicas de época tardoantigua y medieval, las explotaciones vuelven a desarrollarse a partir del siglo XVI y se continúan hasta nuestros días de forma más o menos intensa, como demuestra, para el caso de las canteras marmóreas la documentación manuscrita recopilada por Llordén (1962) ${ }^{2}$. Así ocurrió, por ejemplo, en las canteras almerienses de Macael donde en la década de los 60 de nuestro siglo se destruyeron los frentes de explotación y el poblado romanos, según Canto (1977-78: 171s.).

\footnotetext{
1. Evidentemente tales afloramientos pétreos continúan asimismo en ámbitos de las actuales provincias andaluzas de Granada y Córdoba, donde destaca el centro de Cabra, al que nos referiremos más adelante.

2. Aparte de ese uso abundante en tierras malagueñas, podemos apreciar que su empleo superaba tales límites locales, ya que tenemos constatada la presencia de mármoles de Mijas, por ejemplo, a lo largo del siglo XVIII en los trabajos de remodelación del palacio de San Telmo, en Sevilla; cfr. Falcón 1991: 126 (en 1725), 168 (a partir de 1786), junto a otras piedras de Morón, Estepa y el Puerto de Santa María.
} 


\section{LA LOCALIZACIÓN DE LAS CANTERAS DE ÉPOCA ROMANA}

\subsection{Los mármoles blancos}

Para el caso de los mármoles malagueños sí han existido propuestas concretas de localización de algunas canteras antiguas. Así, se han identificado restos de trabajo que podrían ser de época romana -indudables para Cisneros ${ }^{3}$ - en la denominada cantera de Ardalejos (fig. $1, n^{\circ} 3$ ), en la vertiente norte de la sierra de Mijas, en el término de Alhaurín el Grande, que constituye una de las zonas de mejor accesibilidad dentro de los frentes localizables actualmente en conexión con la salida del material hacia el río Guadalhorce. En el lugar no aparecen huellas del uso de cuñas y sí sólo de picos y cinceles, quedando aún algunos bloques abandonados en uno de los frentes. No obstante, falta en realidad un estudio arqueológico del sitio, para valorar de forma correcta la extensión de la explotación e intentar fijar su cronología que, desde esa falta real de datos indudables, pudo corresponder también a época antigua, a pesar de nuestras dudas anteriores (Loza 1984-85: 132s.)

De cualquier modo la diversidad de las variantes marmóreas testimoniadas en los materiales arqueológicos apuntan a que serían varios los frentes explotados en época antigua en este sector de la actual provincia malagueña. Por ello la localización del resto de las canteras antiguas - considerando como tal la de Ardalejosdebieron coincidir seguramente con algunas de las canteras modernas, ya que la explotación del mármol de estas sierras comienza de nuevo a ponerse en funcionamiento desde el siglo XVI hasta nuestros días de forma ininterrumpida y en diferentes sectores.

También Gozalbes (1986: 241 y mapa en 242, reproducido en nuestra fig. 2) ha indicado la existencia de explotaciones antiguas de mármol en el cortijo Almendral (fig. 1, $\mathrm{n}^{\circ} 1$; fig. 2), dentro del término municipal de Alhaurín de la Torre, en un área de la falda meridional de la sierra de los Espartales; se trata de una pequeña elevación situada al norte de la sierra de Mijas, y en cuya falda septentrional se situaba la antigua Cartima. Precisamente en las tierras de este cortijo, en cuya edificación estuvo reutilizado el pedestal CIL II, 1946, quiso situar erróneamente Rodríguez de Berlanga (1902: 378ss. y 1903: 173) la ciudad de Iluro ${ }^{4}$; sin embargo, no quedan trazas de un yacimiento arqueológico que pueda ser identificado como los restos de una ciudad romana, que pudiera justificar al menos la explotación marmórea a gran escala ${ }^{5}$, y sólo se conoce como procedente de este lugar un epígrafe funerario (CIL II, 5487), perfectamente adscribible a un asentamiento rural.

Gozalbes (1986: 241) indicaba: "Pensamos que en el cortijo del Almendral hubo un taller de labra de productos extraídos de una cercana y antiquísima cantera, hoy apenas apreciable entre matas y rastrojos".

Aunque hay afloramientos de mármoles blancos en ese lugar (correspondientes asimismo al complejo Alpujárride, al que corresponden las sierras de Mijas y Alpujala-Blanca), no hemos logrado identificar restos de explotación, por lo que, si ésta existió, no tuvo mucha importancia, en especial por las características

3. 1988: 24, pero realmente no aporta una base concreta para ello; así, dice: "Aunque no se puede afirmar que estos restos sean los dejados en la antigüedad, ya que falta un estudio de ellos, no ofrece dudas la explotación en época romana de este paraje, como se puede constatar en el capítulo III de este estudio". Sin embargo, en ese lugar del trabajo sólo se vuelve a expresar la misma idea genérica, sin aportar otras razones: “...aunque no se puede afirmar la romanidad de estos restos de extracción, no ofrece dudas la explotación de este lugar en la antigüedad, si bien es necesario un estudio arqueológico de este sector marmorífero". (Cisneros 1988: 101). Por otro lado, este autor sólo identifica dos piezas elaboradas en el mármol concreto de Ardalejos, la escultura de niño y ave de la villa de Torrox-Costa y un pedestal de Barbesula (Cisneros 1988: $\mathrm{n}^{\circ} 31$ y $\mathrm{n}^{\circ}$ 65, respectivamente).

4. Por el contrario su localización en el entorno de Álora es ya indicada por Hübner en CIL II, pág. 246.

5. También dice Gozalbes (1986: 285, nota 35): “...en un rastreo del lugar sólo pudimos comprobar la existencia de cerámica medieval". 
del mármol que se documenta en superficie, poco compacto e inadecuado para una explotación a gran escala, sobre todo en contraposición a los afloramientos de la vecina y más importante sierra de Mijas.

Precisamente en las sierras de Mijas y Alpujala localizamos hasta siete grandes sectores de explotación de época moderna (Loza, Beltrán 1990: 19ss., con base en Loza 1985: 39ss.): tres en la vertiente meridional de la sierra de Mijas (canteras de Osunilla, el Puerto y San Antonio) (fig. 1, nº 4, 5 y 6); uno en la vertiente norte (cantera del arroyo de las Zorreras, en Alhaurín de la Torre) (fig. $1, \mathrm{n}^{\circ} 2$ ) -teniendo en cuenta que también en esa vertiente se sitúa la cantera de Ardalejos (fig. $1, n^{\circ} 3$ ), en Alhaurín el Grande, con su problemática ya planteada-; y, finalmente, tres sectores en la parte oriental de la sierra Blanca, las canteras del cerro de Juan Pérez (fig. 1, n⿳9 9), en Monda, y de La Albuqueria y la Loma del Algarrobo (fig. 1, nº 7 y 8), en Coín.

De visu las principales diferencias se observan entre los mármoles de las dos sierras, Mijas y Blanca, y se ha llegado a diferenciar los mármoles de Mijas y de Coín, como hace Mayer (1992: 18 y bibliografía), pero de los resultados de los análisis petrográficos Cisneros (1988: 24s.) estableció cinco zonas de canteras antiguas diferentes, que deben considerarse, pues: Mijas, Alhaurín de la Torre, Alhaurín el Grande, Coín y Monda ${ }^{6}$.

\subsection{Las calizas blancas y blanco-rojizas}

Para las explotaciones de caliza blanca y blanco-rojiza sólo se han aportado localizaciones genéricas. En primer lugar, las más abundantes calizas blanco-rojizas, denominadas generalmente como "mármol rojizo del Torcal", aunque se tratan de diversas variantes de caliza oolítica con nódulos blancos y cemento rojo, que se documentan en buena parte del surco intrabético malagueño, con núcleos principales en la sierra del Torcal (Atencia 1987: 227s.) y en la sierra de Cañete ${ }^{7}$ (cf. figs. 1, A y B; fig. 2). En segundo lugar, las calizas blancas se explotarían asimismo en la zona del Torcal antequerano, aunque la localización es sólo genérica (Canto 1977-78: 181; Atencia 1988: 228). Desde el punto de vista espacial, y a pesar de esa amplitud territorial comentada, el núcleo principal se sitúa por lo conocido hasta ahora en la zona de la sierra del Torcal, en el centro de ese surco intrabético citado.

\section{ANÁLISIS DE LOS MATERIALES ELABORADOS EN LOS MARMORA MALAGUEÑOS. LA CRONOLOGÍA DE LA EXPLOTACIÓN Y USO}

Debemos, pues, pasar al análisis de las piezas arqueológicas identificadas en estos marmora para poder establecer conclusiones o hipótesis más ajustadas del período de explotación y empleo. En este aspecto observaremos también una elemental diferencia entre los materiales de ambos sectores, ya que se han realizado análisis petrográficos como base para la identificación de muchos de los mármoles malagueños y también se han identificado petrográficamente en sectores fuera del ámbito territorial malagueño, lo que ofrece un panorama más rico. Por el contrario falta este tipo de análisis en el caso de las calizas malagueñas, con sólo la excepción de una placa de caliza blanca aparecida en la excavación sevillana de c/ Argote de Molina. No obstante, a la problemática concreta de los marmora localizados en este lugar (no sólo calizas blancas

6. De forma más pormenorizada en Lapuente, Cisneros, Ortiga 1988.

7. Así también lo manifiesta Gozalbes (1986: 241 ss. y mapa en 242) al destacar el sector antequerano y otro en torno a Cañete y Teba; pero no corresponde a esta serie la cantera que cita de Pinedilla (fig. 2) -muy cercana a la ciudad de Singilia Barba-, ya que se trata de una importante cantera de arenisca; además, erróneamente afirma -quizás confundiéndolas con las canteras de caliza blanca antequeranas-que "...de esta cantera [de Pinedilla] proceden grandes cantidades de piedra utilizada en Itálica". Cf. las correctas referencias de Atencia 1987: 228. 
antequeranas, sino también mármoles blancos malagueños) -a la que no queremos renunciar por su incidencia para fijar el inicio de la explotación romana de ambos tipos de piedra-nos referiremos de forma más extensa en un apéndice, ya que afecta a cuestiones del urbanismo romano de Hispalis y disturbarían nuestra exposición actual.

\subsection{Explotación y uso de los mármoles blancos}

En el estudio ya citado y presentado al II Congreso de Historia Antigua de Málaga hemos llevado a cabo un catálogo de piezas elaboradas en mármol blanco de las sierras de Mijas y Alpujala-Blanca, al que remitimos, permitiéndonos ahora sólo realizar una breve referencia a esas series, desarrolladas con base en nuestros trabajos, donde recogimos una cincuentena de piezas, arquitectónicas, escultóricas, de soportes epigráficos y varios; eran de procedencia en su mayoría local, de los actuales territorios malagueños, con la excepción de un pedestal de estatua de Algeciras (Loza, Beltrán 1990: 30ss.). La procedencia concreta correspondía, sobre todo, a ciudades que se situaban en el entorno de los propios afloramientos marmóreos; de forma destacada, Suel (Fuengirola) y, sobre todo, Malaca (Málaga) y Cartima (Cártama). En segundo lugar, se constata una menor documentación de piezas en otras ciudades como Nescania (Valle de Abdalajís), Singilia Barba (El Castillón, Antequera) o Lacipo (Alechipe, Casares), en función de las facilidades de comunicación con las canteras. Asimismo en el entorno costero malacitano se testimonia un uso abundante en diversas villae, como las de Torreblanca del Sol y Secretario (Fuengirola), Acevedo y Butibamba (Mijas) o Torrox, y-hacia el interior, pero muy cerca de la vertiente norte de la sierra de Mijas- la villa de Fuente del Sol (Alhaurín el Grande).

En estos yacimientos se testimonia el uso de los mármoles blancos malagueños en todos los ámbitos. Entre los materiales arquitectónicos destacan basas y fustes de columnas, capiteles, cornisas, etc.; entre los escultóricos destacan las grandes esculturas de los ámbitos próximos a las explotaciones, especialmente en Malaca y Cartima, aunque asimismo en las villae costeras de Torreblanca y Secretario. Finalmente también se constata su empleo para la elaboración de soportes epigráficos, sobre todo pedestales de estatua y placas, y otros elementos, como, en concreto, placas lisas de recubrimiento o, de manera excepcional, tesellae de mosaicos.

Con posterioridad se amplió esa serie -de forma concreta, para sectores extramalacitanos-sobre todo por Cisneros (1988: 94s.) y, parcialmente, por Lapuente, Cisneros y Ortiga (1988). Especialmente el primero, y aparte de dos piezas dudosas de ámbito malacitano (cfr. Beltrán, Loza e.p., notas 33-35), identifica piezas en la villa de Lecrín (Granada) (dos esculturillas decorativas de las termas), las ciudades de Barbesula (San Roque, Cádiz) (dos pedestales de estatua), de Baelo Claudia (Bolonia, Cádiz) (columna del foro), de Gades (estatua de gran formato) y, finalmente, en la moderna Chiclana (Cádiz) (placa epigráfica). En el ámbito del Guadalquivir destacaba su presencia en las ciudades de Hispalis (un capitel y, al menos, una placa de recubrimiento, cuya problemática analizamos en el Apéndice) y Corduba (altar funerario). Fuera de este ámbito, y asimismo con dudas lógicas (cfr. Beltrán, Loza e.p., nota 38), identificó Cisneros una escultura en Caesaraugusta.

La importancia de tales identificaciones radica no tanto en el número, que no es muy grande, sino en las localizaciones, ya que testimonia la presencia de piezas elaboradas en mármol malagueño en toda la zona costera actual gaditana y en el ámbito del Guadalquivir, al menos en la zona navegable. Asimismo, nosotros hemos ampliado la documentación del mármol blanco en otros materiales de Hispalis y, asimismo, de Italica (vid. Beltrán, Loza e.p., Apéndice). Con seguridad ese número aumentaría de forma significativa si se llevaran a acabo análisis más exhaustivos. 
Finalmente, Vaquerizo y Noguera (1997) han identificado en mármol malagueño ${ }^{8}$ casi una quincena de esculturas decorativas procedentes de la villa de El Ruedo (Almedinilla, Córdoba) (fig. 2), que presentan el interés de la localización interior del yacimiento, en la zona meridional de las actuales campiñas cordobesas, así como demuestran la importancia del mármol malagueño en las producciones escultóricas locales de los talleres béticos en la época altoimperial.

A partir de la datación de toda la producción documentada podemos deducir, en primer lugar, el período de explotación de las canteras. Así, debemos mantener, pues, que la explotación organizada del mármol blanco malagueño debió iniciarse en época julio-claudia, cuando menos poco antes de mediados del siglo I d.C., en concreto a partir de su identificación en el retrato cartimitano de Calígula, que sería elaborado entre los años 37 y 41 d.C., indicándonos el más seguro hito ante quem, aunque ello no quiera decir que la producción no comenzara en época de Augusto, como parece lógico, sobre todo a nivel local.

Como es sabido la monumentalización y marmolización bajo modelos romanos de las ciudades hispanas tiene lugar especialmente a partir de época augústea y a lo largo de la dinastía julio-claudia, incorporándose asimismo como elemento determinante el culto imperial (por ejemplo, Beltrán 1994; Abascal 1996). A ambos fenómenos podríamos referenciar el uso del mármol malagueño en la arquitectura del foro de Baelo (a fines de la dinastía julio-claudia) y del de Hispalis -con las precisiones cronológicas aportadas en el Apéndice-; pero, asimismo en otras ciudades más cercanas se observa un uso monumental del mármol local, en los diversos ámbitos de la arquitectura, la escultura o la epigrafía, como demuestran una cornisa julio-claudia del sector del teatro malacitano, o un togado asimismo de la capital malagueña o la placa marmórea de Lacipo que conmemora la construcción de un recinto de culto imperial en la ciudad asimismo en momentos julio-claudios (Puertas, Rodríguez Oliva 1980: esp. 23ss.), y en cuya edificación quizás se utilizó asimismo este mármol, aunque es un extremo no constatado por ahora.

Fue la época flavia el momento en que la mayor parte de las ciudades del entorno, como la propia Malaca o Lacipo, y también Cartima, Suel o Nescania, accedieron a la municipalidad y ello debió favorecer la expansión del uso y consecuente explotación durante ese período, que enlazaría con el momento de máximo empleo constatado durante el siglo II d.C., al menos por los materiales fechados hasta el momento ${ }^{9}$.

A pesar de que continúa un uso arquitectónico -como demuestran, por ejemplo, los capiteles malacitanos de la segunda mitad del siglo II d.C.-, puede observarse que destacan asimismo las piezas escultóricas y epigráficas, en relación con los renovados programas ornamentales, de carácter tanto público como privado. En efecto, son esculturas (de divinidades, imperiales o de magistrados y prohombres locales) y monumentos epigráficos (sobre todo pedestales) de ámbito público, pero también de ámbito privado, sobre todo en villae, que a partir de este momento empiezan a tener un desarrollo importante de las partes urbanae, con áreas ajardinadas y complejos termales donde destacan las esculturas decorativas o las estatuas-fuentes (Loza 1993; Beltrán 1995: 207ss.). En este sentido es bastante significativo el caso citado de la villa de Almedinilla (Córdoba), donde la mayor parte del rico conjunto escultórico ornamental está elaborado en mármol mijeño, o asimismo la villa granadina de Lecrín, aunque de aquí sólo proceden dos esculturas.

El siglo III d.C., y en concreto sobre todo el final de la dinastía severa -en paralelo, por ejemplo, a lo que ocurre a nivel general de la estatuaria hispana ${ }^{10}$-, marca el final de esa importante producción, y se produciría entonces el abandono de las explotaciones a raíz de los materiales datados entonces; en todo caso su explotación a partir de entonces sería sólo residual.

8. Los análisis fueron realizados por el Laboratorio para el Estudio de Materiales Lapídeos Antiguos (LEMLA) de la Universidad Autónoma de Barcelona.

9. Cf., no obstante, las indicaciones aportadas por Padilla (1999: 278), en el sentido de que “...el último tercio del siglo I y los inicios del III [se convirtió] en la etapa de mayor volumen de la actividad evergética relacionada con las construcciones públicas y, paralelamente, en el momento culminante del consumo del mármol y de la actividad de las canteras béticas".

10. Sobre todo en el ámbito de la escultura decorativa; cf., por ejemplo, Loza 1993; Koppel 1993. 
En relación a la cronología de las explotaciones, si seguimos los resultados de los análisis proporcionados por Cisneros, podrían establecerse algunas hipótesis de los períodos de utilización. Excluyendo algunos materiales visigodos o altomedievales, se identificaron 37 ejemplares romanos elaborados en mármoles malagueños ", con la siguiente proporción y dataciones -más o menos precisas:

1) En primer lugar, sobresalen las 22 piezas documentadas como de la zona de Mijas -casi un 60 por ciento de la producción total identificada-, con dataciones tanto durante el siglo I d.C. como el siglo II d.C., llegando en algunos casos a los comienzos de la centuria siguiente.

2) A continuación se identifican 6 piezas de mármol de Coín, destacando grandes esculturas de carácter público -de Cartima y Malaca-, datadas durante el siglo II d.C., a las que sólo puede unirse un capitel de Hispalis, no fechado.

3) Siguen 4 piezas identificadas como de mármol de Alhaurín de la Torre, que, con excepción de un pedestal cartimitano de época flavia, asimismo se datan en el siglo II d.C.

4) A continuación 3 ejemplares identificados como de mármol de Monda, que incluyen la columna del foro de Baelo, de época claudia-neroniana, la placa sevillana de c/Argote de Molina -para la que proponemos una fecha más probable de al menos el siglo I d.C.--, y la escultura de Archidona de finales del siglo I d.C.

5) Finalmente, como procedentes de la cantera de Alhaurín el Grande (Ardalejos) se identifican sólo 2 piezas, ambas del siglo II d.C., una de ellas un pedestal barbesulano datado entre los años 147-161 d.C.

$\mathrm{Si}$ concedemos valor representativo a tales proporciones y dataciones ${ }^{12}$, podríamos pensar que durante el siglo I d.C. se ponen en funcionamiento exclusivamente las explotaciones de Mijas (en la vertiente meridional de la sierra de Mijas) y de Monda (en sierra Blanca), y que durante el siglo II d.C. se amplió la producción de la de Mijas, la de Monda se debió abandonar, siendo sustituida por la de Coín en ese sector oriental de sierra Blanca, y, finalmente, se abrieron también en esa centuria las de Alhaurín de la Torre y Alhaurín el Grande, en la parte norte de sierra de Mijas, aunque sin eclipsar la producción de las dos anteriores. En efecto, serían las producciones de la vertiente sur de la sierra de Mijas (seguidas bastante después por las de Coín) las que abastecieron el mercado en su mayoría durante los siglos I-II d.C.

\subsection{Explotación y uso de las calizas blancas y blanco-rojizas}

Ese rico panorama que ofrece la investigación en el caso de los mármoles no es comparable al que podemos deducir de las explotaciones de calizas del norte de la provincia, ya que falta un catálogo exhaustivo de los materiales elaborados y, además, del origen concreto de cada uno de los ejemplares que conocemos ${ }^{13}$. Por otro lado, sólo en una ocasión una pieza de caliza blanca aparece reconocida por Cisneros ${ }^{14}$-dentro

11. Cisneros 1988: 94 ss. Se trata de una fuente-caño de Cártama, de la segunda mitad del s. VI d.C.-fines del s. VII d.C., y un soporte epigráfico de Mesas de Villaverde datado a fines del s. X d.C.-comienzos del s. XI d.C. (Cisneros 1988: 135). Diferente es la proporción que se ofrece en Lapuente, Cisneros, Ortiga 1988: 273 (cuadro: 25 de Mijas, 4 de Coín, 4 de Alhaurín de la Torre, 3 de Monda y 2 de Alhaurín el Grande), pero seguimos las ofrecidas por Cisneros en la obra citada.

12. Obviamos la consideración de la datación del siglo II a.C. de Sevilla (edificio de sillares de la c/Argote de Molina), como se dirá en el Apéndice.

13. La situación es menos problemática en el caso de los soportes epigráficos, ya que conocemos ahora repertorios actualizados de los conventus Cordubensis (CIL $\Pi^{2} / 7$ ) y-especialmente para el caso de las calizas blancas y blanco-rojizas malagueñas-Astigitanus $\left(\mathrm{CIL} \mathrm{II}^{2} / 5\right)$, identificándose los materiales en que se elaboran, pero lógicamente no se puede hacer todavía la identificación concreta de los sectores de donde proceden.

14. Cisneros 1989-90: 128. No incluye, sin embargo, la pieza en su estudio general (Cisneros 1988: 72s.), en el que hace referencia sólo a la caliza blanca antequerana por los comentarios previos y genéricos de Canto (1977-78: 181), pero sin identificar ningún material de esta procedencia en sus 202 piezas analizadas. No obstante, debe tenerse en cuenta que la representación general de los marmora usados en la Hispania romana a partir de los resultados de esta obra es parcial, ya que del total de las 202 piezas 
de la problemática particular ya aludida de los materiales pétreos de c/ Argote de Molina (Sevilla)-, con lo que no contamos con esa fundamental fuente de información sobre la distribución de sus productos a nivel regional.

Algunos datos apuntan a que la situación es más compleja de lo que podemos pensar hoy día. Así, ya Grünhagen (1978: 196s.) identificó la caliza blanca antequerana en Munigua y Canto (1977-78: 181) afirmó en relación a ese mismo material que: "...en Itálica hay muchas piezas de esta caliza, entre ellas las dos basas de M. Lucrecio Juliano, del teatro, y varios capiteles antiguos". Ambos pedestales de estatua, de los que se conservan sólo la parte central paralelepipédica -ya que fueron elaborados en tres partes separadas-, presentan una inscripción repetida dedicada por la ciudad a aquel caballero en el reinado conjunto de Septimio Severo, Caracala y Geta (entre 209-211 d.C. en concreto), aunque fueron reutilizados después en la escena del teatro italicense (González 1991: 51ss., n³79, fig. 209).

Aparte de esta referencia explícita, el problema estriba en la dificultad de identificación de estos tipos de marmora fuera de su entorno de explotación sin recurrir a una analítica concreta, por la similitud visual que pueden tener con otras piedras similares de otras regiones, de las que incluso en el peor de los casos se desconoce su explotación en época antigua. Otro problema lo supone la abundancia de afloramientos susceptibles de explotación en una amplia zona geográfica, como ocurre con las calizas rojas en el norte malagueño, que incluso se continúan en la zona meridional de Córdoba, con las similares calizas rojas de Cabra (Segura 1988: 112ss.), o en Granada, como documenta su uso en soportes epigráficos de Ilurco o Iliberris (CIL $\mathrm{II}^{2} / 5$ : 164ss.).

No obstante, hemos llevado a cabo un primer acercamiento -que tampoco pretende ser exhaustivo ${ }^{15}$ en los ámbitos malacitanos sobre el uso de tales marmora, para poder establecer algunas hipótesis que futuros trabajos corroborarán, así como para constrastarlas ahora, en segundo lugar, con el modelo de los mármoles malagueños.

Como parecería lógico es mayor el número de piezas elaboradas en calizas rojas -por la mayor abundancia de afloramientos- y asimismo los lugares donde se testimonian. Así, se documenta el uso de las calizas rojas en ciudades del interior (fig. 1), como-desde el oeste al este-Acinipo, Sabora, el oppidum ignotum del cortijo del Tajo en Teba, Nescania, Singilia Barba, Antikaria, Osqua, Aratispi o Vlisi, y sus respectivos territoria, y, más hacia la costa, también en Cartima o la propia Malaca. Aunque no de una forma tan abundante y extensa, también se documentan piezas elaboradas en calizas blancas en algunas de las ciudades citadas como Osqua, el oppidum ignotum del cortijo del Tajo en Teba, Sabora o, sobre todo, Singilia Barba.

Corroboran las conclusiones aportadas por Atencia derivadas de su estudio sobre el poblamiento romano de la Depresión de Antequera, al afirmar que la producción de las calizas blanco-rojizas era "...consistente sobre todo en pedestales de estatuas, arae, columnas y basas... y otros elementos de construcción de cierta suntuosidad", mientras que en caliza blanca “....se labraron algunos pedestales y placas molduradas de recubrimiento" ( Atencia 1986: 227 y 228, respectivamente).

También estas últimas calizas blancas tienen un aprovechamiento en el campo arquitectónico, según demuestran, por ejemplo, testimonios aislados, como los capiteles citados de Italica o una interesante cornisa procedente de Sabora (Cañete), elaborada en el siglo II d.C. (Atencia 1987: 143ss.), pero también panoramas más completos, como el que ofrece la excavación de Singilia Barba, donde sus excavadores documentan

identificadas, 23 corresponden a Regina (Casas de Reina, Badajoz) (n's 72-94) y 103 a Augusta Emerita (Mérida, Badajoz) ( $n^{\circ}$ s 95-197), por lo que al resto peninsular sólo restan 76 identificaciones (Cisneros 1988: 87s.).

15. Así, destacamos, entre otros: Serrano, De Luque, Rodríguez Oliva 1975; Sillières 1978; Serrano, Atencia 1981; Atencia 1981; Atencia 1982; Beltrán 1982-83; Rodríguez Oliva, Atencia 1983; Rodríguez Oliva 1984; Atencia 1984-85; Rodríguez Oliva 1985; Atencia 1986; Atencia 1987a; Atencia 1987 b; Atencia 1988; Beltrán 1988; Serrano, Rodríguez Oliva 1988; Atencia, De Luque 1989-90; Serrano, Atencia, Rodríguez Oliva 1991-92; Atencia 1993; Romero 1993-94. En lo que respecta a los frecuentes soportes epigráficos documentados se recopilan ahora en, el ya citado, CIL II²/5. 
el empleo importante de la caliza blanca "...en capiteles, molduras, placas de revestimiento y solerías..." (Serrano, Atencia, De Luque, Rodríguez Oliva 1991: 278). Sin embargo, en función de lo publicado hasta ahora, destaca su uso en el campo de la epigrafía, tanto para placas, como especialmente para altares y pedestales honorarios, sobre todo datados a lo largo del siglo II d.C. Así lo han reconocido los importantes trabajos de Atencia referidos, por ejemplo, a esa producción epigráfica en Sabora, en Antikaria y, especialmente, en Singilia Barba (cf., ahora, $\mathrm{CIL} \mathrm{II}^{2} / 5$ ).

Aunque algunos de estos monumentos estaban decorados con relieves, como el altar de Osqua decorado con escenas de sacrificio y una representación imperial -posiblemente de fines de los julio-claudios ${ }^{16}$-, el pedestal, posiblemente anticariense o en todo caso singilense, con sendas Victorias en relieve ornamentando los laterales (Beltrán 1982-83), u otro pedestal dedicado a un Fabio Fabiano en el cortijo del Tajo y decorado con erotes guirnaldóforos (Rodríguez Oliva 1984: 437ss.; CIL II²/ 5: 857) -ambos del siglo II d.C.-, debe reconocerse que este tipo de caliza no tuvo un empleo frecuente en la escultura, a pesar de su calidad.

El uso de las calizas blanco-rojizas es bastante similar al de las blancas, tanto por un empleo muy importante en el campo de los soportes epigráficos, especialmente altares y pedestales -incluso superior cuantitativamente por la mayor abundancia de afloramientos en todo el interior de la actual provincia malagueña-, como por su escaso empleo en el campo de la escultura. No obstante, debemos destacar una pieza escultórica que puede ser significativa, ya que parece testimoniar la importancia que se concedía a su vistoso colorido, que recordaba el aspecto del "pavonazzetto" de Asia Menor. Así, se utilizó este tipo de caliza en la escultura de un Attis procedente del teatro de Malaca (Baena 1984: 73, $n^{\circ}$ 13, lám. 13,2), ya que en este caso su similitud con el mármol frigio debía servir como rememoranza del similar origen del representado, en un mecanismo bastante usual en la escultura romana imperial para el uso de mármoles coloreados exóticos (Schneider 1986).

El hecho cuenta además con el evidente paralelo de varias esculturas ornamentales elaboradas en las similares calizas rojas de Cabra, como son un Attis de Lucena y varios hermae de jardín de Puente Genil, Nueva Carteya y la misma Córdoba capital ${ }^{17}$, piezas éstas que cuando son de importación normalmente aparecen elaboradas en mármoles coloreados, como el "giallo antico" tunecino o asimismo el "pavonazzetto" frigio. Se tratarían de ejemplos de ese fenómeno de "marmora de sustitución", que por su similitud sustituyen a mármoles foráneos, de mayor prestigio y coste ${ }^{18}$.

Por el contrario, estas calizas rojas-y a diferencia de las blancas-tuvieron un enorme empleo documentado en el ámbito arquitectónico, aunque-como manifestaba Atencia (1986: 227)-como materiales constructivos de cierta suntuosidad, especialmente placas ornamentales, fustes y capiteles, donde precisamente resaltaría su aspecto coloreado. El ejemplo más evidente nos lo ofrece la ciudad de Singilia Barba -que ya hemos referido (Serrano, Atencia, De Luque, Rodríguez Oliva 1991)-, porque las importantes excavaciones arqueológicas llevadas a cabo en el yacimiento nos ofrecen el más completo panorama del uso de estos marmora en una ciudad próxima a los centros de producción y de florecimiento urbano durante el Alto Imperio. Así, observamos que las calizas rojas se emplean en grandes bloques como escalinatas de acceso a edículas de la zona del foro, o en pedestales junto a los de caliza blanca en esa misma zona forense, o en fustes de columnas de un edificio doméstico, o en las más abundantes placas de recubrimiento.

El panorama que manifiesta este yacimiento urbano no debe ser muy diferente del que tuvieron otras ciudades de estas zonas interiores malagueñas en época altoimperial, donde las calizas locales constituirían los marmora más empleados, por su calidad y abundancia, unido a las dificultades de transporte para los marmora foráneos. Pero incluso en lugares alejados del entorno geográfico del surco intrabético se documenta

16. Baena 1981: 73, aunque lo da como mármol grisáceo de cantera local y del siglo II d.C.

17. Segura 1988: 121, aunque habría que recurrir a una más completa analítica para establecer la procedencia exacta, dada las cercanías de las explotaciones al ámbito malagueño y los lugares de descubrimiento, en su mayoría en la campiñas meridionales cordobesas. Ya había documentado su uso en Munigua Grünhagen (1978: 296s.).

18. Sobre ese fenómeno en nuestro marco bético trata ahora Padilla (1999). 
el uso de calizas rojas, como ocurre en Cartima o en la cercana villa de la "Fuente del Sol" (en Alhaurín el Grande) -con el uso de fustes lisos y, en el segundo caso, capiteles esquemáticos ${ }^{19}$-, en Malaca o en la villa de Río Verde (Marbella). No obstante, desconocemos donde se situarían las explotaciones de estas calizas concretas, si eran traídas desde el interior o se obtenían en zonas más cercanas, como en el valle del Guadalhorce.

Este panorama es similar al que ofrecen las calizas rojas de Cabra -y no podía ser menos, puesto que presentan unas características pétreas similares y parecidas condiciones de explotación-; como ha destacado Segura hubo un importante uso tanto en elementos arquitectónicos y de ornamentación, como en soportes epigráficos, mientras que en la escultura sólo se documentan las estatuillas ornamentales ya citadas (Segura 1988: 119ss.).

En lo que respecta a la cronología que aportan los materiales elaborados en calizas malagueñas -algunos datados con bastante precisión por los epígrafes imperiales que presentan-, se concentran mayoritariamente en el siglo II d.C., llegando hasta el período severiano, y remontando su empleo abundante a la época flavia -sin duda asimismo en relación con los procesos de monumentalización urbana asociados a las correspondientes obtenciones de estatutos municipales, pero asimismo en el ámbito rural de villae, como por ejemplo testimonian algunos hermae de época flavia.

En este caso podemos aportar mayores precisiones cronológicas para el inicio de la explotación y uso con base en algunos epígrafes conservados y bien datados. Así, debemos citar sendos pedestales de Antikaria dedicados a Tiberio, en el 25 d.C. (CIL II/5, 747), y a Julia Augusta, entre el 14-29 d.C. (CIL II²/ 5, 748) y un soporte funerario de Singilia Barba datado en época de Augusto (CIL II ${ }^{2} / 5,811$ ). Nos corroboran, pues, la temprana puesta en funcionamiento de estas explotaciones, al menos en los casos citados, en función especialmente de los programas de monumentalización pública, pero que asimismo afectaban a necesidades privadas, como testimonia la lápida también citada.

El margen cronológico sería entonces más amplio que el dado para las ya referidas cercanas canteras de caliza roja de Cabra, que se iniciarían desde época flavia según Segura (1988: 119), momento en que en la Bética se desarrolla especialmente el gusto por los mármoles coloreados, como apuntó Grünhagen (1978: 290ss.) en su estudio de los marmora de Munigua. No obstante, la propia existencia de afloramientos de tales calizas de buena calidad y la dificultad del transporte de otros marmora de ámbito regional -especialmente los mármoles blancos de la costa malagueña ${ }^{20}$ - determinarían la preponderancia del empleo de las calizas blancas y, sobre todo, blanco-rojizas en las ciudades y territorios de las zonas interiores de la actual provincia malagueña como principales marmora de carácter local, especialmente en los ámbitos de la arquitectura y los soportes epigráficos.

\section{APÉNDICE: ¿EXPLOTACIÓN EN EL S. II A.C.?}

En relación al momento de inicio de la explotación de estos materiales pétreos malagueños nos encontramos con un interesante problema, que requiere una atención especial, pero que se hace más complejo porque sólo se cuenta con referencias parciales o indirectas, poco explícitas. Así, se han identificado los marmora malagueños (al menos una placa elaborada en mármol de la zona de Monda y otra en caliza blanca de

19. Para la primera, Rodríguez Oliva (1985 b), para la segunda, Andérica (1982), aunque en este caso nos encontramos con una caliza algo diferente de la de la zona norte, ya que presenta nódulos blancos de mayor tamaño y en algún caso casi recristalizados.

20. Beltrán, Loza e.p. En cualquier caso el mármol malagueño está documentado de forma esporádica, como ocurre en la placa conmemorativa de la reforma de la basílica del foro de Singilia Barba a mediados del s. II d.C. (CIL II²/ 5: 794). Diverso sería el caso de los marmora foráneos, bien documentados -al menos desde una perspectiva cualitativa-, pero que respondían a diferentes criterios no estrictamente económicos, sino asimismo de prestigio y tradición. 
Antequera) en elementos decorativos del denominado como "edificio de sillares" de la c/ Argote de Molina (Sevilla) -excavado por J. Campos en 1983 y considerado un sector del foro republicano de la ciudad ${ }^{21}$-. Es Cisneros (1988: 95) quien desarrolla en realidad tales cuestiones referidas a las placas ornamentales, adscribiéndolas a fases del edificio entre mediados del siglo II a.C. y fines del siglo I a.C.

La cuestión se complica porque esa temprana fecha-sobre todo, la cronología alta de la segunda centuriadada al uso del mármol malagueño en Hispalis es compartida además por otros tipos de marmora hispanos. Así, de la serie de materiales pétreos analizados -“...casi una treintena, que sin duda debieron revestir el edificio...", según Campos (1986: 22, nota 20)-Cisneros (1988: 98, nota 40, 137) identifica los siguientes: tres placas elaboradas en calizas de Alconera (Badajoz), una más en mármol portugués de Vila Viçosa y otras dos de Estremoz, correspondiendo una de ellas a una moldura decorada. A ellas debería unirse -según Cisneros- la placa identificada como caliza blanca antequerana, pero a la que este autor no se refiere en su estudio citado de 1988, sino en un artículo dado a la luz poco después (Cisneros 1989-90: 128, nota 26), indicando incluso el $n^{\circ}$ de inventario de la excavación ( $\left.n^{\circ} 1997\right)$, por lo que hemos de considerar que la ausencia de referencia en la obra general anterior se debió simplemente a un error.

Pero surgen nuevas interrogantes, ya que los materiales pétreos no se referencian en el catálogo -incompleto- de materiales de la memoria de excavación correspondiente (Campos 1986), por lo que desconocemos realmente si todos los fragmentos pétreos correspondían a una sola fase o a qué niveles concretos deben adscribirse. Sólo en un caso ocurre lo contrario, correspondiendo a la moldura elaborada en mármol de Estremoz ( $n^{\circ}$ inventario 3347), que Campos (1986: 64 y 70, fig. 37) adscribe a los materiales del nivel 19 (fechado en la segunda mitad del siglo II a.C.). Al menos esa datación plantea el problema de la temprana cronología a la que nos referimos.

En resumen, no sabemos, en realidad, si el resto de materiales pétreos aducidos por Cisneros (y entre ellos las placas de materiales malacitanos) tenían similar procedencia estratigráfica, a pesar de esa referencia textual de su excavador antes indicada, que las adscribe todas a la decoración del edificio republicano, y que se amortizaría hacia mediados del s. I a.C., según Campos. Es más, si nos fiamos del número de inventario 1997 correspondiente a la placa de caliza blanca antequerana, ésta debería situarse en los niveles 11 ó 12, ique se datarían entre la segunda mitad del siglo IV d.C. y la primera mitad del siglo V d.C.! (Campos 1986: 17 y 69).

Como desconocemos los números de inventario del resto de los materiales pétreos no podemos saber realmente en qué estratos aparecieron, pero nos parece que, frente a consideraciones globales, como la efectuada por Cisneros, deberían tenerse en cuenta algunas consideraciones:

- No todos los marmora proceden del mismo nivel y, por tanto, no deben necesariamente corresponder a la misma edificación y cronología de uso y abandono.

- A partir de los dos únicos fragmentos de los que conocemos su ubicación estratigráfica se constataría un arco cronológico desde la segunda mitad del siglo II a.C. (mármol de Estremoz) hasta la primera mitad del siglo $\mathrm{V}$ d.C. (caliza antequerana).

- No existe constancia de que los marmora correspondieran a la ornamentación del "edificio de sillares", de cuya cronología, por otra parte, podría dudarse mediante una revisión crítica de algunos de sus fundamentos, como se dirá luego. No obstante, para el caso de la moldura de mármol de Estremoz sí se afirma de forma concreta una datación de la segunda mitad del s. II a.C.

Cisneros, considerando en principio todo el lote de fecha republicana, ya expresaba lógicamente su extrañeza, puesto que, por los datos que conocemos -y que creemos pertinentes-, en general estas explotaciones

21. Campos 1986: esp. 15ss. Sobre la interpretación de este sector y el correspondiente "edificio de sillares", cf. Campos 1989: 245ss.; Campos, González 1987: 123ss. Campos sostiene que el "edificio de sillares" corresponde a la monumentalización del foro republicano del siglo II a.C., que será abandonado a fines del siglo siguiente (con la construcción de un nuevo espacio forense en la zona de la plaza de la Alfalfa), reestructurándose finalmente todo el sector en época trajano-adrianea, en relación con la edificación del cercano conjunto arquitectónico de c/ Mármoles. 
citadas no se inician hasta el siglo I d.C., sobre todo en la cantidad y organización suficientes para sustentar esos procesos de comercialización que llevarían a la Hispalis romana marmora de Estremoz, Alconera y Málaga. Este autor, no obstante, abría una posibilidad a esa cronología alta, con el paralelo en el empleo durante el primer tercio del siglo I a.C. del mármol de Cabezo Gordo (Campo de Cartagena, Murcia) en un pavimento del yacimiento del Castillet (Murcia) ${ }^{22}$. Sin embargo, ello no es comparable, ya que la diversidad de materiales testimoniados en la excavación sevillana y la distancia que separan los diversos centros de Hispalis indicaría, como se ha dicho, la existencia de complejos sistemas de producción y comercialización en época tardorrepublicana, algo que no nos parece factible.

Además, hemos de citar otras dudas sobre la interpretación cronológica y estratigráfica de la excavación sevillana, en concreto en lo referido al citado "edificio de sillares". Es de destacar, en primer lugar, la reciente crítica llevada a cabo por S. Ordóñez (1998: 34ss.) a las bases cronológicas expuestas por Campos, por el erróneo valor que según él se concedieron a las cerámicas ibéricas de bandas, a los materiales cerámicos de barniz negro o a las ánforas vinarias Dressel 1, concluyendo que "...existen serias dificultades para remontar la cronología de la reconstrucción de este edificio hispalense - o al menos la de su ornamentación marmóreamás allá de esa fecha de comienzos del siglo I d.C." (Ordóñez 1998: 35). Con similar contundencia se expresa A. Padilla (1999: 274s.) en referencia a la negativa del uso de esa panoplia de marmora en el edificio sevillano en el s. II a.C.

Aunque no es éste el momento para plantear una crítica exhaustiva de la interpretación de la secuencia estratigráfica de la excavación (cfr. figs. 3 y 4) -para la que faltan además datos fundamentales-discutible es, por ejemplo, la asociación que se hace entre las estructuras de sillares y el suelo de opus signinum, considerado como pavimento original del edificio, ya que, aparte de no existir relación material evidente entre ambos elementos, supondría una cimentación de los muros -apoyados sobre un "encarche de piedras"de menos de $0,50 \mathrm{~m}$., algo poco probable en paramentos de tales características. $\mathrm{O}$ asimismo que el "edificio de sillares" presentara una superficie decreciente en las paredes interiores, como se afirma con base en la colocación de las tres líneas de sillares documentados en dos de los extremos de la cuadrícula de sondeo. Quizás ambas circunstancias podrían interpretarse como resultado de que nos encontramos con potentes cimentaciones de sillares que cortarían depósitos anteriores -entre ellos el citado suelo de opus signinum-, asentándose sobre una estructura de opus caementicium preexistente, posiblemente sí de época republicana ${ }^{23}$, aunque ello es simplemente una intuición indemostrable.

De cualquier modo esa "reinterpretación" no explicaría la presencia de materiales pétreos en el nivel 19 -si ello es así, y al menos se dice en el caso de la moldura de mármol portugués ya citada-. Es evidente la simple y lógica imposibilidad de que esa moldura de mármol de Estremoz, aparecida en el nivel 19 (Campos 1986: 16ss. y esp. figs. 3-4, de los perfiles, y 12, de la planta, y fot. 3) -un depósito de relleno entre el "encarche de piedras" y el suelo de opus signinum (los pretendidos cimentación y pavimento del "edificio de sillares", como se dijo)-, pudiera pertenecer a la decoración del edificio, ya que en ese caso el fragmento habría sido recuperado en un nivel coetáneo cronológicamente -e incluso inferior desde el punto de vista estratigráfico-del suelo de opus signinum, considerado como el pavimento original del edificio republicano que supuestamente decoraban los marmora. Si el resto de marmora tuviera esa misma procedencia-excepto

22. Cisneros 1988: 137ss., aunque realmente apunta que “...son precisos más documentos de este tipo para aseverar, sin márgenes de error, esta hipotesis".

23. La fecha de la cercana estructura arquitectónica de c/Mármoles, que no debió corresponder a un templo, como se afirmaba (cf. especialmente Rodríguez Temiño 1991), y los materiales epigráficos (Campos, González 1987) nos indican una importante transformación de este sector urbano durante el s. II d.C., que fue interpretada por el mismo Campos mediante la secuencia de abandono del foro republicano en época augustea, con un uso no constatado, y su documentación como plaza cubierta de albero a principios del s. II d.C., en conexión con el "templo" de c/Mármoles (Campos 1986; Campos 1993). Sobre la hipotesis de la localización del teatro en un ámbito próximo a este sector Ordoñez 1998 b. 
la placa de caliza antequerana, que podemos suponer que no la tiene, según lo ya dicho sobre su número de inventario-, habría que suponerles similares dificultades.

El problema del temprano uso en Hispalis de las producciones de Málaga (Monda y Antequera), junto a las de Alconera, Estremoz y Vila Viçosa se diluye, pues, dentro de esa problemática apuntada, y su empleo debe corresponder a momentos imperiales.

\section{BIBLIOGRAFÍA CITADA}

ABASCAL, J.M. (1996): "Programas epigráficos augusteos en Hispania", AAC, 7: 45-82.

ANDÉRICA, J.R. (1982): "Una villa romana en Alhaurín el Grande (Málaga): la Fuente del Sol”, Actas del I Congreso Andaluz de Estudios Clásicos, Jaén: 119-125.

ATENCIA, R. (1981): "La problemática de la epigrafía antikariense", Arqueología de Andalucía Oriental: Siete Estudios, Málaga: 133ss.

__ (1982): "De epigrafía nescaniense", Baetica, 5: 115-120.

_- (1984-85): "El epígrafe singiliense de G. Vallio Maxumiano, reencontrado", Mainake, VI-VII: 177-186.

_- (1986): El poblamiento rural y urbano de época romano en la Depresión de Antequera, Málaga (Tesis Doctoral inédita).

_ (1987a): "El poblamiento antiguo en la Depresión de Antequera", Actas del II Congreso Andaluz de Estudios Clásicos, vol. II, Málaga: 205-229.

- (1987 b): "Sobre los restos arqueológicos del cortijo de la Colada (Cañete la Real, Málaga)", Baetica, 10: 139-159.

(1988): La ciudad romana de Singilia Barba (Antequera, Málaga), Málaga.

(1993): “Proc. Aug. Ad. 'Fal. Veget.' Sobre un epígrafe gemelo de CIL II 2029 (P. Magnius Rufus Magonianus)", Estudios dedicados a Alberto Balil. In memoriam, Málaga: 113-132.

ATENCIA, R.; DE LUQUE, A. (1989-90): “A propósito de dos pedestales con inscripciones honorarias de Singilia Barba, Antequera (Málaga)", Mainake, XI-XII: 171ss.

BAENA, L. (1981): "El ara romana del Museo Arqueológico Municipal de Antequera", Arqueología de Andalucía Oriental: Siete Estudios, Málaga: 73ss.

- (1984): Catálogo de las Esculturas Romanas del Museo de Málaga, Málaga.

BELTRÁN, J. (1982-83): "Un monumento romano dedicado a la Victoria en el Museo de Antequera", Mainake, IV-V: 227-236.

(1988): Las arae de la Baetica, Málaga.

(1994): "Análisis arqueológico de modelos urbanos en ciudades hispanorromanas durante el Alto Imperio", Kolaios. Publicaciones Ocasionales, 3, Sevilla: 59-80.

(1995): "La incorporación de los modelos griegos por las élites romanas en ámbito privado. Una aproximación arqueológica", E. Falqué, F. Gascó, eds., Graecia capta. De la conquista de Grecia a la helenización de Roma, Huelva: 207-232.

BELTRÁN, J.; LOZA, M.L. (e.p.): "El comercio de los mármoles blancos malagueños durante el Alto Imperio Romano", F. Wulf, G. Cruz, eds., II Congreso de Historia Antigua de Málaga. Comercio y comerciantes en la Antigüedad, Málaga, en prensa.

CAMPOS, J. (1986): Excavaciones arqueológicas en la ciudad de Sevilla, Sevilla.

- (1989): "Estructura urbana de la Colonia Iulia Romula Hispalis en época republicana", Habis, 20: 245-262.

(1993): "La estructura urbana de la colonia Iulia Romula Hispalis en época imperial", $A A C, 4: 181-219$.

CAMPOS, J.; GONZÁLEZ, J. (1987): “Los foros de Hispalis Colonia Romula”, AEspA, 60: 123-158. 
CANTO, A.M. (1977-78): “Avances sobre la explotación del mármol en la España romana”, $A E s p A, 50-51$ : 165-189.

CISNEROS, M. (1988): Mármoles hispanos: su empleo en la España Romana, Zaragoza.

- (1989-90): "Sobre la explotación de calizas en el sur de España en época romana: canteras de Gádor (Almería), Atarfe (Granada), Antequera (Málaga) y Cabra (Córdoba)", Caesaraugusta, 66-67: 123ss. FALCÓN, T. (1991): El palacio de San Telmo, Sevilla.

GONZÁLEZ, J. (1991): Corpus de inscripciones latinas de Andalucía. II: Sevilla. II. La Vega (Itálica), Sevilla $(=$ CILA 3).

GOZALBES, C. (1986): Las vías romanas de Málaga, Madrid.

GRÜNHAGEN, W. (1978): "Farbiger Marmor aus Munigua”, MM, 19: 290-306.

KOPPEL, E. (1993): "Die Skulpturenausstattung römischer Villen auf der Iberischen Halbinsel”, en W. Trillmich y otros, Hispania Romana. Die Denkmäler der Römerzeit, Mainz: 193-203.

LAPUENTE, M.P.; CISNEROS, M.; ORTIGA, M. (1988): "Contribución a la identificación de las explotaciones de mármoles españoles empleados en la Antigüedad", $N A H, 30: 255 \mathrm{ss}$.

LEÓN, P. (1995): Esculturas de Itálica, Sevilla.

LLORDÉN, A.: Arquitectos y canteros malagueños, Ávila.

LOZA, M.L. (1984-85): "Notas sobre la explotación del mármol blanco de la sierra de Mijas en época romana", Mainake, VI-VII: 131-136.

LOZA, M.L. (1985): La explotación del mármol blanco de la sierra de Mijas en época romana. Las canteras, Málaga (Memoria Licenciatura inédita).

LOZA, M.L. (1992): La decoración escultórica de fuentes en Hispania, Málaga.

LOZA, M.L.; BELTRÁN, J. (1990): La explotación del mármol blanco de la sierra de Mijas en época romana. Estudio de los materiales arquitectónicos, escultóricos y epigráficos, Monografías de Faventia 10, Bellaterra.

MAYER, M. (1992): "L'exploitation des ressources lapidaires en Hispanie", Le Marbre dans l'Antiquité (= Les Dossiers d'Archéologie, 173), Paris.

MAYER, M.; RODÁ, I. (1998): "The use of marble and decorative stone in Roman Baetica", S. Keay, ed., The Archeology of Early Roman Baetica, JRA Suppl. Ser. 29, Portsmouth: 217-234.

ORDÓNEEZ, S. (1998): Primeros pasos de la Sevilla romana (Siglos I A.C. - I D.C.), Sevilla.

- (1998 b): "Edificios de espectáculos en Hispalis: una propuesta de interpretación de CIL II 1193", Habis, 29: 141-156.

PADILLA, A. (1999): "Consideraciones en torno a la explotación del mármol en la Bética durante los siglos I-II", Habis, 30: 271-282.

PASTOR, M. (1987): "Vestigios arqueológicos de época romana en 'La Camila' (Archidona, Málaga)", Actas del II Congreso Andaluz de Estudios Clásicos, vol. II, Málaga: 247-259.

PUERTAS, R.; RODRÍGUEZ OLIVA, P. (1980): Estudios sobre la ciudad romana de Lacipo (Casares, Málaga), Studia Archaeologica, 64, Valladolid. .

RODÁ, I. (1997): "Los mármoles de Itálica. Su comercio y origen”, A. Caballos; P. León, eds., Itálica MMCC. Actas de las Jornadas del 2200 Aniversario de la Fundación de Itálica, Sevilla: 155-180.

RODRÍGUEZ DE BERLANGA, M. (1902): “Estudios Epigráficos. ¿Alhaurín Iluro?”, Revista de la Asociación Artístico Arqueológica Barcelonesa, vol. III, 29: 378ss.

(1903): Catálogo del Museo Loringiano, Málaga.

RODRÍGUEZ OLIVA, P. (1981): "Notas sobre algunas esculturas romanas de la zona oriental del conventus de Gades", Baetica, 4: 79-88.

(1984): "La Antigüedad", Málaga. II. Historia, Granada: 437ss. 
(1985a): "Un nuevo testimonio de los hermes-retratos en la Baetica: la pilastra hermaica de Osqua", Baetica, 8: 165-190.

— (1985 b): "Investigaciones arqueológicas del marqués de Valdeflores en Cártama (1751-1752)", Cártama en su historia, Málaga: 59-70.

RODRÍGUEZ OLIVA, P.; ATENCIA, R. (1983): "Estelas saborenses", BSAA, 49: 151 ss.

RODRÍGUEZ TEMIÑO, I. (1991): "Algunas cuestiones sobre el urbanismo de Hispalis en época republicana", Habis, 22: 157-175.

ROMERO, M. (1993-94): “La necrópolis de las Maravillas. Bobadilla. Málaga”, Mainake, XV-XVI: 195-222.

SCHNEIDER, R.M. (1986): Bunte Barbaren, Worms.

SEGURA, M.L. (1988): La ciudad ibero-romana de Igabrum (Cabra, Córdoba), Córdoba.

SERRANO, E.; ATENCIA, R (1981): Inscripciones latinas del Museo de Málaga, Madrid.

SERRANO, E.; ATENCIA, R.; DE LUQUE, A.; RODRÍGUEZ OLIVA, P. (1991): "Informe de las excavaciones arqueológicas realizadas en la ciudad romana de Singilia Barba (Antequera), en la campaña de 1989", AAA/1989. II. Actividades Sistemáticas, Sevilla: 269-79.

SERRANO, E.; ATENCIA, R.; RODRÍGUEZ OLIVA, P. (1991-92): "Novedades epigráficas de Singilia Barba", Mainake, XIII-XIV: 171-203.

SERRANO, E; DE LUQUE, A.; RODRÍGUEZ OLIVA, P. (1975): “Varia arqueológica malacitana”, Jábega, 11: 44s.

SERRANO, E.; RODRÍGUEZ OLIVA, P. (1988): "Tres nuevas inscripciones de Singilia Barba (El Castillón, Antequera, Málaga)", Baetica, 11: 237-256.

SILLIÈRES, P. (1978): “Nouvelles inscriptions de Singilia Barba (El Castillón, Antequera, Málaga)”, MCV, 14: $465 \mathrm{ss}$

VAQUERIZO, D.; NOGUERA, J.M. (1997): La villa de El Ruedo, Almedinilla (Córdoba). Decoración escultórica e interpretación, Murcia. 


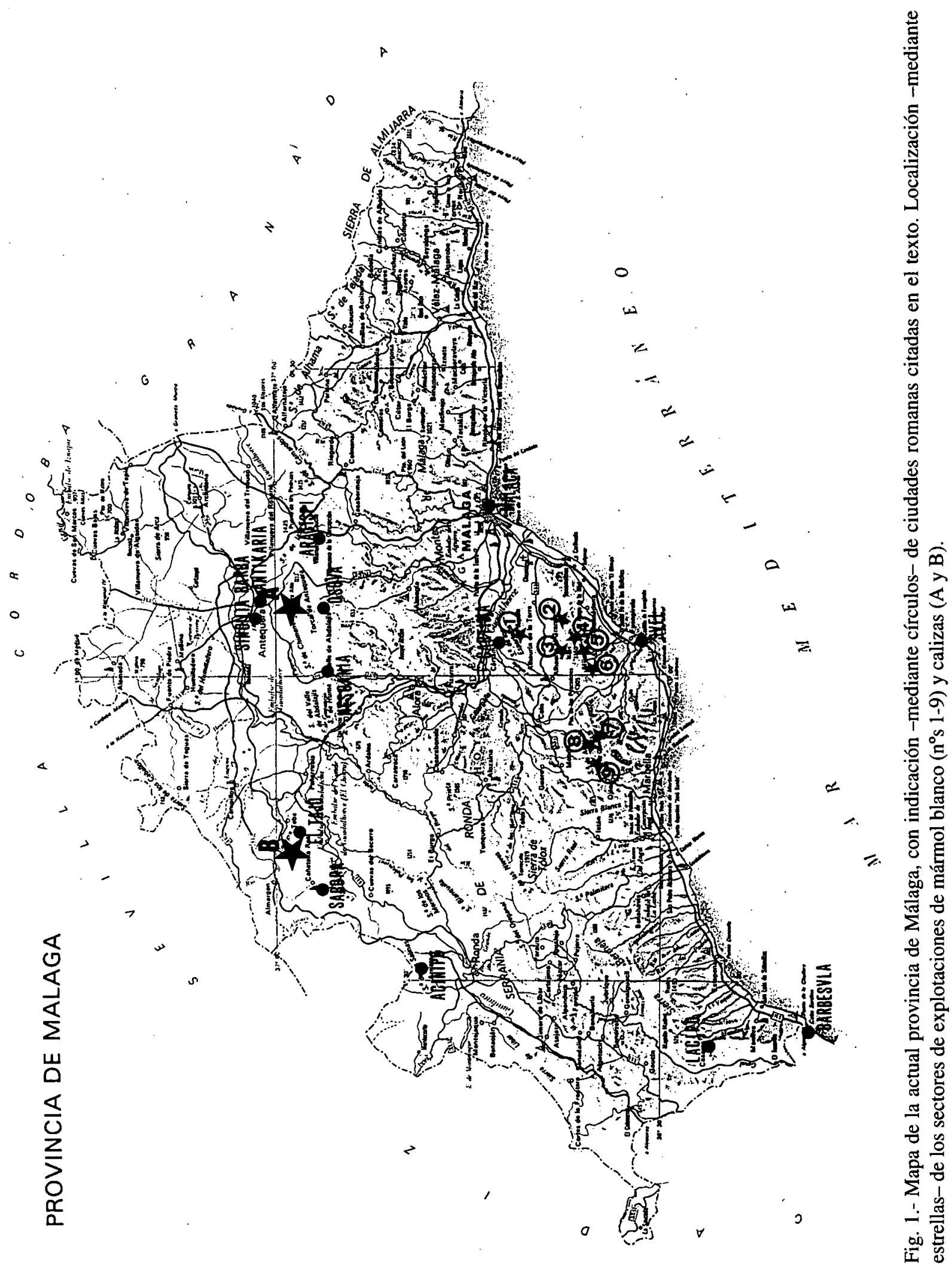




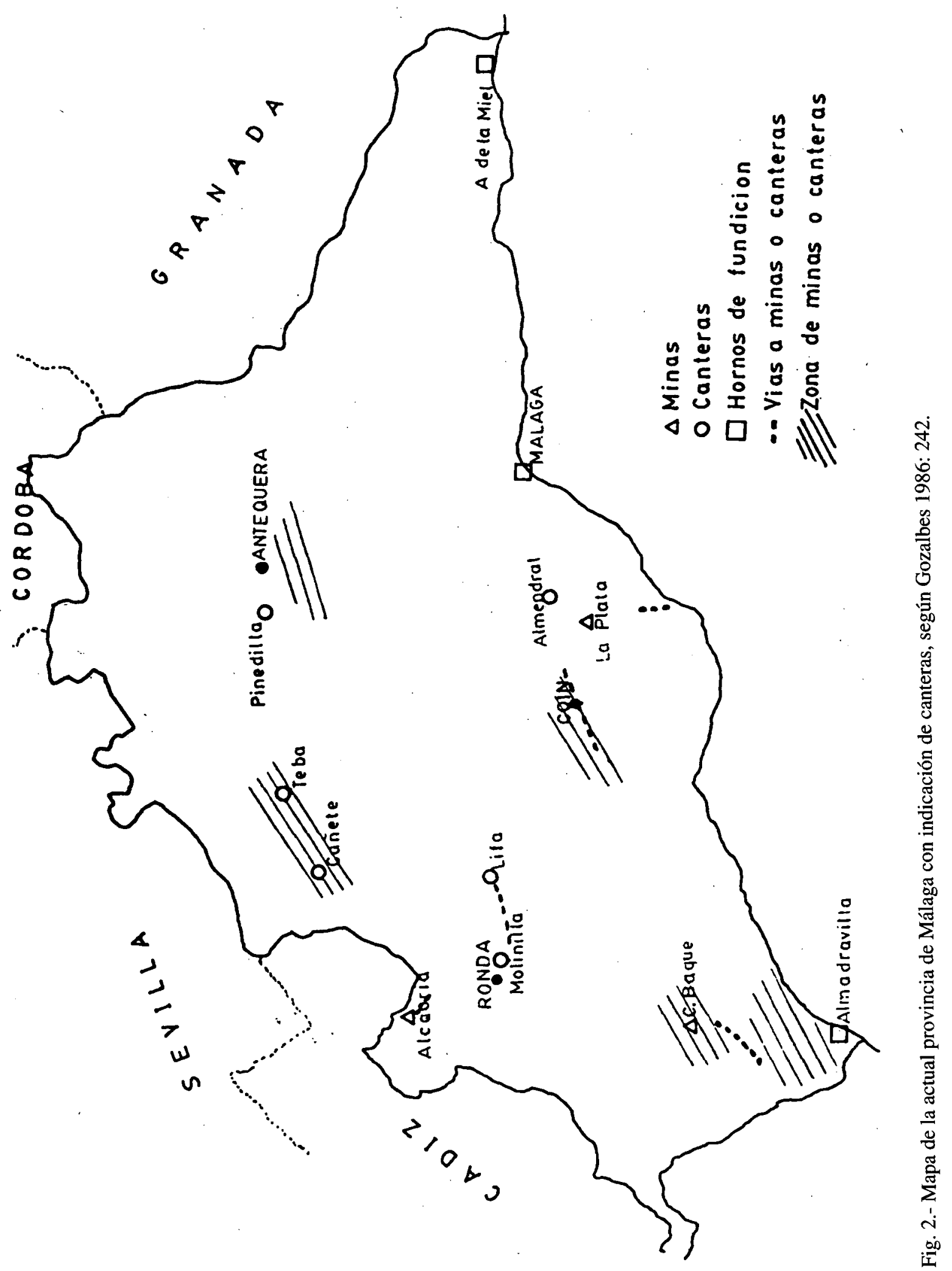



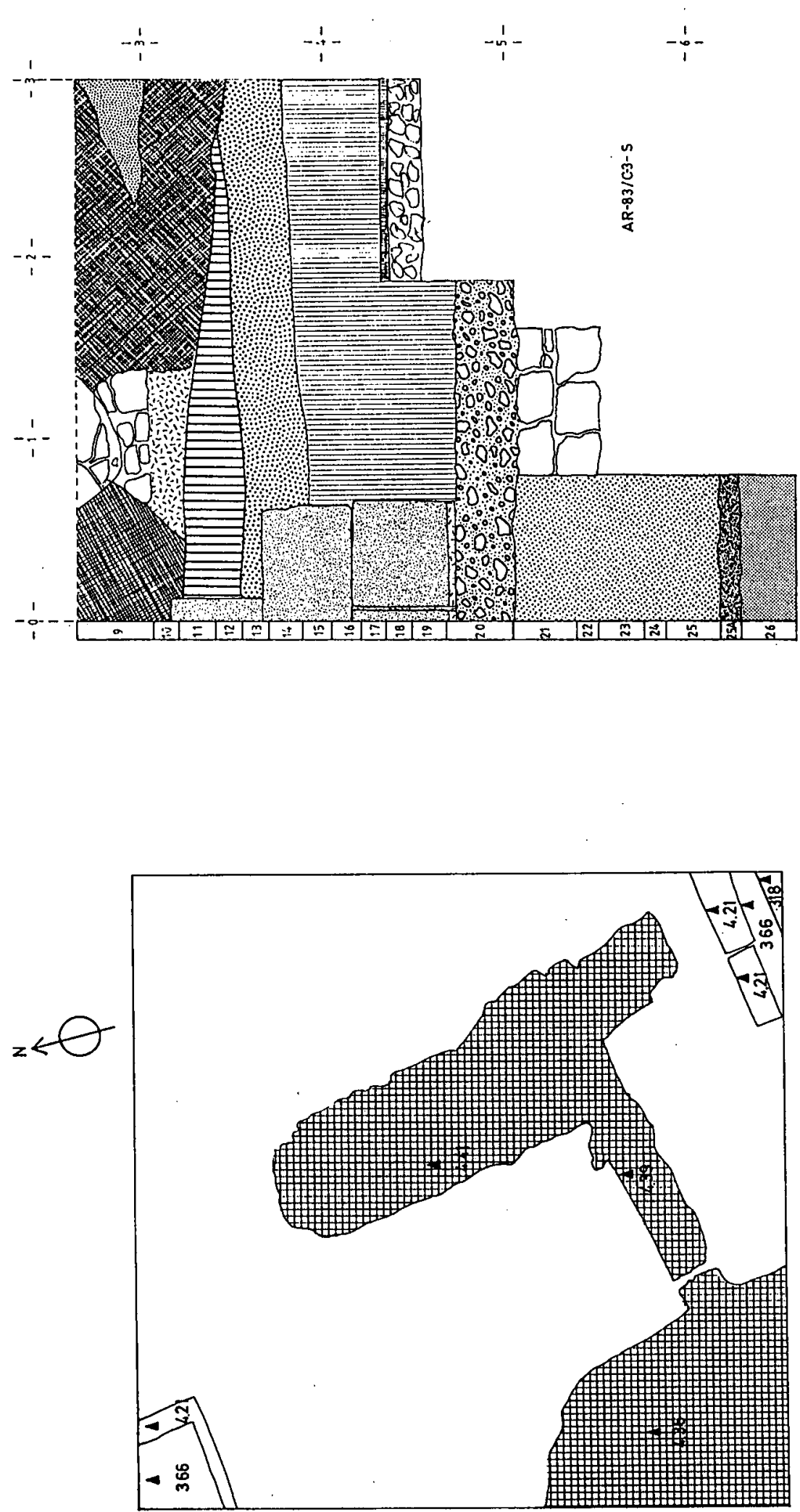

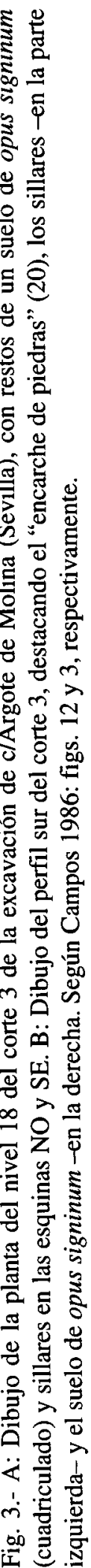



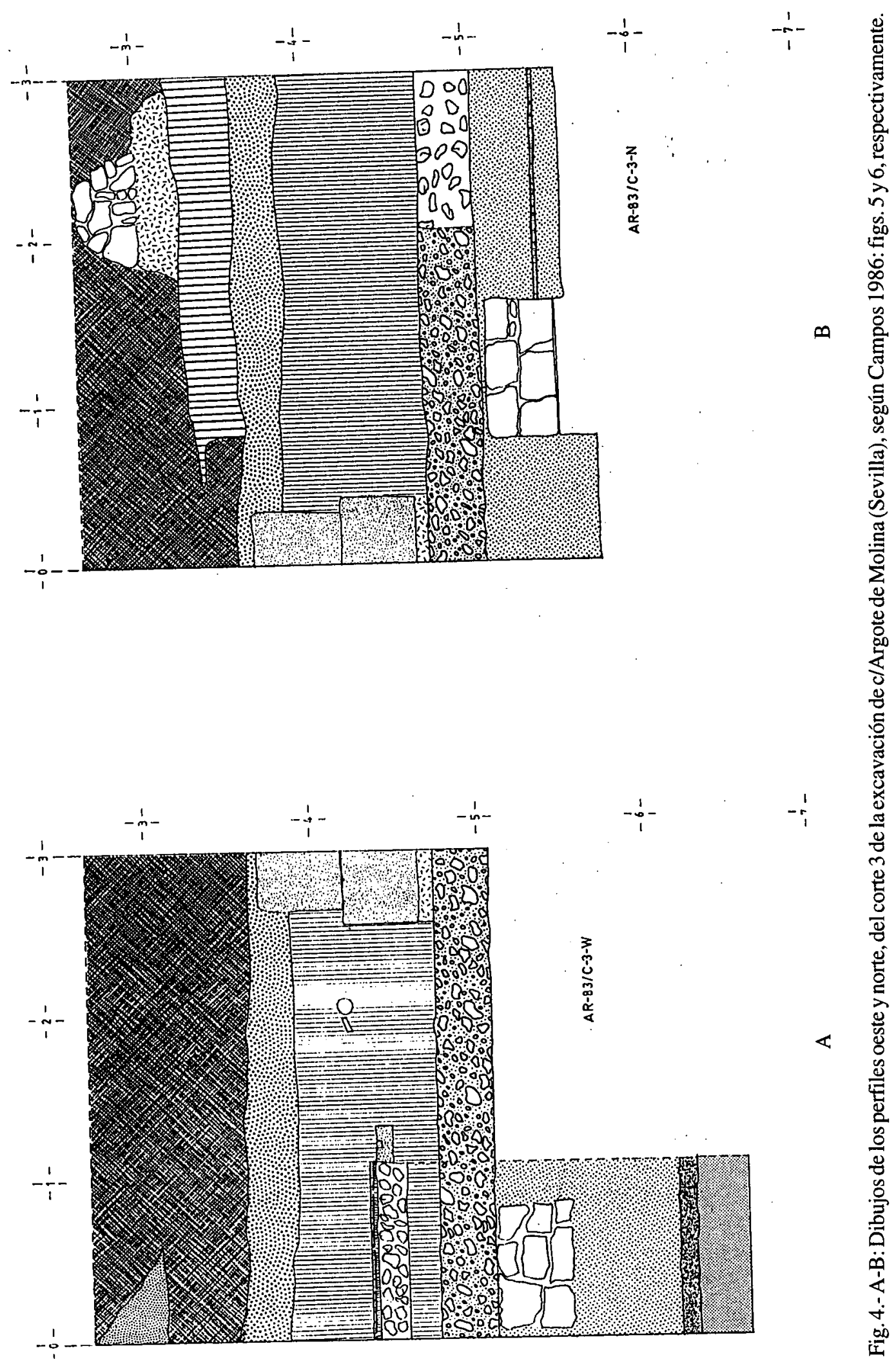